\title{
TWO-DIMENSIONAL CONSOLIDATION OF A CROSS-ANISOTROPIC CLAY DEPOSIT
}

\author{
By Yukitoshi MURAKAMI*
}

\begin{abstract}
The two-dimensional consolidation of a cross-anisotropic clay deposit is theoretically examined. Based on the Biot theory ${ }^{1)}(1955)$, the solutions of the consolidation settlement and of the excess pore water pressure are presented, although certain constraint relationships of elastic parameters are imposed. Some results of calculation are shown and the characteristics of consolidation are discussed to assess the influence of soil anisotropy.
\end{abstract}

\section{INTRODUCTION}

Natural clay deposits exhibit some degree of cross-anisotropy since clay platelets have been oriented in a sub-horizontal arrangement throughout their sedimentation and subsequent self-weight consolidation. There is a need to take the influence of soil cross-anisotropy into consideration when estimating the rates of consolidation settlement and of excess pore water pressure dissipation in reference to the construction of a structure on such a clay deposit. However, the studies of consolidation which take account of the soil anisotropy are very few, although the basic theory has been already developed by Biot ${ }^{1)}$ in 1955 . This fact may be referred to the complicated mathematical treatment. Among these, Murakami ${ }^{4)}$ (1982) has theoretically examined the consolidation of a clay deposit with cross-anisotropic permeability and has pointed out that the anisotropy of permeability has great influence on the rate of consolidation and the Mandel-Cryer effect. Tamura ${ }^{5)}$ (1983) has discussed the consolidation in a cross-anisotropic ground with sand drains which generate only a radial flow of pore water. The former study gives attention only to the anisotropy of permeability, while the latter study only to the anisotropy of soil deformation. It may be so considered that no analytical investigations of consolidation which account for the anisotropy of both permeability and deformation have been yet published although they are, in fact, coupled by reason of deriving from the anisotropy of soil structure.

This Note treats the two-dimensional consolidation of a cross-anisotropic clay deposit analytically in order to assess the effect of soil anisotropy on the consolidation characteristics, considering the anisotropy of both permeability and soil deformation.

* Member of JSCE, Dr. Eng., Associate Professor, Department of Civil Engineering, University of Yamanashi (Takeda 4 Chome, Kofu, Yamanashi) 


\section{PROBLEM FORMULATION AND SOLUTIONS}

The equations of equilibrium and continuity under a plane strain condition are expressed in terms of the horizontal and the vertical displacements of soil skeleton $\mathrm{u}, \mathrm{w}$ and the excess pore water pressure $\sigma$ as follows :

$$
\begin{aligned}
& C_{1} \frac{\partial^{2} u}{\partial x^{2}}+C_{2} \frac{\partial^{2} u}{\partial z^{2}}+C_{3} \frac{\partial^{2} w}{\partial x \partial z}=\frac{C_{2}}{G_{v}} \cdot \frac{\partial \sigma}{\partial x} \\
& C_{2} \frac{\partial^{2} w}{\partial x^{2}}+C_{4} \frac{\partial^{2} w}{\partial z^{2}}+C_{3} \frac{\partial^{2} u}{\partial x \partial z}=\frac{C_{2}}{G_{v}} \cdot \frac{\partial \sigma}{\partial z} \\
& \frac{\partial}{\partial t}\left(\frac{\partial u}{\partial x}+\frac{\partial w}{\partial z}\right)=\frac{1}{\gamma_{w}}\left(k_{x} \frac{\partial^{2} \sigma}{\partial x^{2}}+k_{z} \frac{\partial^{2} \sigma}{\partial z^{2}}\right)
\end{aligned}
$$

in which

$x, z=$ coordinates

$t=$ time

$\gamma_{w}=$ weight of water in unit volume

$G_{v}=$ shear modulus in vertical plane

$C_{i}(i=1,2,3,4)=$ auxiliary elastic constants (see Appendix)

$k_{x}, k_{z}=$ horizontal and vertical coefficients of permeability.

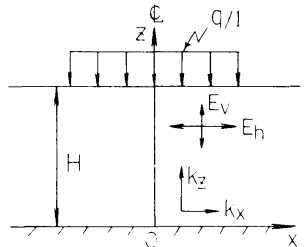

Fig. 1 Strip loading on an anisotropic clay deposit.

The consolidation problem in the case of a uniform strip loading of q per unit area on the surface of a clay deposit, as shown in Fig. 1, can be solved on the basis of Eqs. (1), if the constraint relationship:

$C_{1}=C_{4}=C_{2}+C_{3}$

is satisfied, which signifies that $\partial \sigma_{x}^{\prime} / \partial \varepsilon_{x}=\partial \sigma_{z}^{\prime} / \partial \varepsilon_{z}$ (which does not necessarily mean isotropy) and $G_{v}=$ $\left(1-\mu_{h}-n \mu_{v}\right) E_{v} / 2\left(1-\mu_{h}-2 n \mu_{v}^{2}\right)$ (for $E_{v}, \quad n, \mu_{h}$ and $\mu_{v}$ see Appendix), where

$\sigma_{i}^{\prime}, \varepsilon_{i}=$ effective stress and normal strain in $i$-direction respectively.

The method of analysis is identical with the one described in the author's preceding paper ${ }^{6)}(1976)$. Then, the solutions of $\sigma$ and $w$ are obtained in the following forms:

$$
\begin{aligned}
& \sigma / q=(2 / \pi) \int_{0}^{\infty} \sum_{\alpha} S_{\alpha}(\xi, Z) \exp \left(-K T_{v}\right) \cos (X \xi) d \xi \ldots \ldots \ldots \ldots \ldots \ldots \ldots \ldots \ldots \\
& \left(w-w_{t \rightarrow \infty}\right) G_{v} / H q=(2 / \pi) \int_{0}^{\infty} \sum_{\alpha} W_{\alpha}(\xi, Z) \exp \left(-K T_{v}\right) \cos (X \xi) d \xi
\end{aligned}
$$

in which

$$
\begin{aligned}
& S_{\alpha}(\xi, Z)=\left(C_{1} / C_{2}\right)\left(B_{1} \cos (\alpha Z)+B_{2} \sin (\alpha Z)\right)+B_{3} \cosh (\xi Z)+B_{4} \sinh (\xi Z) \\
& \begin{aligned}
W_{\alpha}(\xi, Z)= & \alpha B_{1} \sin (\alpha Z) /\left(\alpha^{2}+\xi^{2}\right)-\alpha B_{2} \cos (\alpha Z) /\left(\alpha^{2}+\xi^{2}\right)+\left(1-C_{3} \beta / C_{2}\right) B_{3} Z \\
& \quad \cdot \cosh (\xi Z) / 2+\left(1-C_{3} \beta / C_{2}\right) B_{4} Z \sinh (\xi Z) / 2+B_{5} \xi \sinh (\xi Z)+B_{6} \xi \cosh (\xi Z)
\end{aligned} \\
& \begin{aligned}
& K= c_{h} \xi^{2}+c_{v} \alpha^{2} \\
& X= x / H \quad(H: \text { thickness of clay deposit }) \\
& Z=z / H
\end{aligned} \\
& T_{v}=c_{v} t / H^{2} \quad: \text { time factor } \\
& c_{h}=\left(C_{1} / C_{2}\right) k_{x} G_{v} / \gamma_{w} \quad: \text { horizontal coefficient of consolidation } \\
& c_{v}=\left(C_{1} / C_{2}\right) k_{z} G_{v} / \gamma_{w} \quad: \text { vertical coefficient of consolidation } \\
& \beta=\left(C_{1} / C_{2}\right) \cdot\left(c_{h}-c_{v}\right) \xi^{2} /\left(c_{h} \xi^{2}+c_{v} \alpha^{2}\right)
\end{aligned}
$$

$B_{i}(i=1,2, \cdots, 6)$ in Eqs. (2) and (3) are arbitrary constants of integration, to be determined from the boundary conditions of the problem, and $w_{t \rightarrow \infty}$ is the elastic solution of vertical displacement, which has been already solved, for example, by Milovic et $\mathrm{al}^{3)}$. (1970). 


\section{SOME RESULTS OF CALCULATION}

Based on Eqs. (2) and (3), some calculation has been carried out for the case that the base of a clay deposit is smooth and impervious, considering the fact that the anisotropy of deformation is not remarkable for a normally consolidated clay ${ }^{2}$.

Figs. 2 and 3 show the effect of the anisotropy of permeability on the degree of settlement $U_{s}\left(=\left(w-w_{t=0}\right) /\left(w_{t \rightarrow \infty}-w_{t=0}\right)\right)$ at the center of a strip load. It can be seen that the consolidation proceeds smoothly on account of the horizontal drainage of excess pore water when the horizontal permeability is high as compared with the vertical one. However, it should be noted that when $b / H$ ( $b:$ half width of load, $H:$ thickness of clay deposit) is more than 4 the effect of the horizontal permeability on the progress of consolidation disappears.

Fig. 4 indicates the time factor $T_{v}$-degree of settlement $U_{s}$ relationships about three clay deposits, one of which has a realistic cross-anisotropic property and the others have the isotropic property of deformation. They are intended to be nearly equivalent in respect to Poisson's ratio, considering the fact that the characteristics of consolidation are greatly influenced by Poisson's ratio of soil skeleton (Yamaguchi et $\mathbf{a l}^{6}{ }^{6}$. , 1976). It can be found that the relation curves are close each other, particularly in the range of $U_{s} \leq 0.5$. This matter means that if the anisotropy of deformation, one of whose indices is

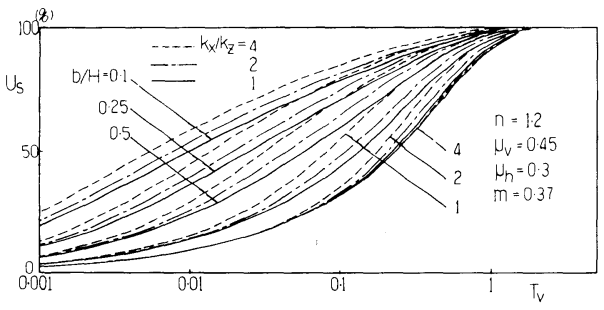

Fig. 2 Time factor $T_{v}$-degree of settlement $U_{s}$ relationships for various anisotropic permeabilities.
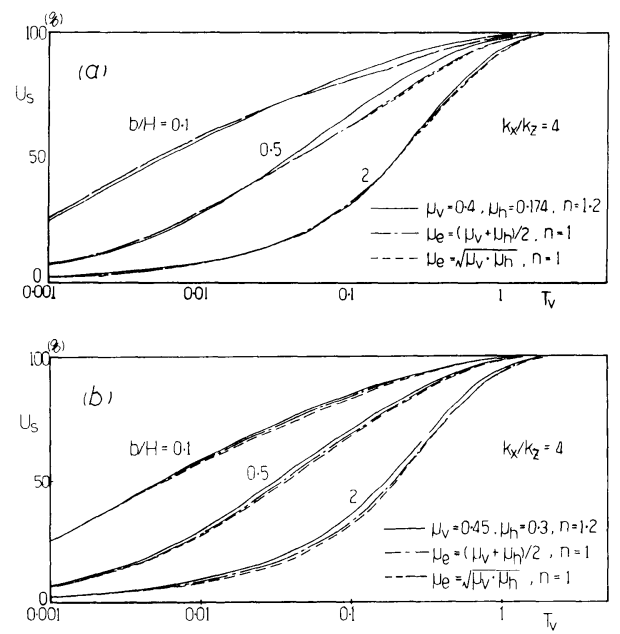

Fig. 4 Relationships of time factor-degree of settlement about three clay deposits.

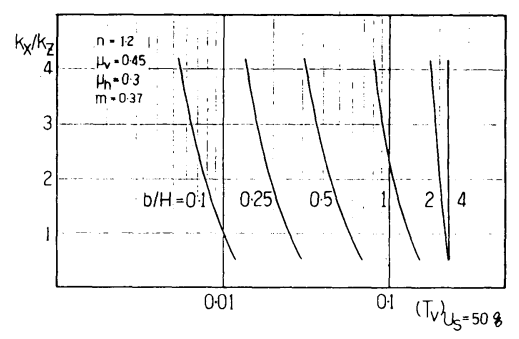

Fig. 3 Effect of the anisotropy of permeability on the time factor corresponding to $U_{s}=50 \%$.

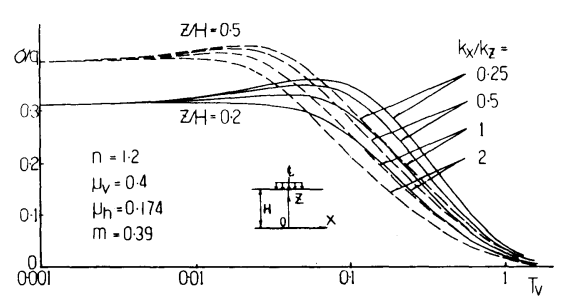

Fig. 5 Time history of excess pore water pressure at some points.

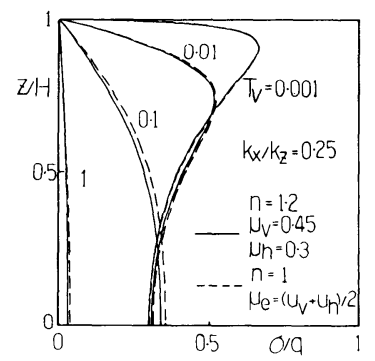

Fig. 6 Time history of excess pore water pressure along a center line of load. 
$n=E_{h} / E_{v}$, is low in the degree then the rate of consolidation settlement can be approximately estimated by substituting the clay deposit which has the equivalent isotropic property of deformation.

Figs. 5 and 6 show the time history of excess pore water pressure at some specified points and along the center line of a load, respectively. It can be seen that when the horizontal permeability is lower than the vertical one the transient rise of excess pore water pressure (due to the change in the mean total stress with time), that is, the Mandel-Cryer effect is remarkable and this phenomenon occurs strikingly in the neighborhood of an impervious base. The characteristics are substantially the same as seen in the consolidation of a clay deposit with cross-anisotropic permeability (Murakami, 1982).

\section{CONCLUDING REMARKS}

The two-dimensional consolidation of a cross-anisotropic clay deposit has been analytically studied. As a result, the following findings can be drawn from the investigation.

(1) The anisotropy of permeability has greater influence on the rates of consolidation settlement and of excess pore water pressure dissipation rather than the anisotropy of soil deformation.

(2) If the anisotropy of deformation, one of whose indices is $n=E_{h} / E_{v}$, is low in the degree, the rate of consolidation settlement can be approximately estimated by substituting the clay deposit which has the equivalent isotropic property of deformation.

\section{APPENDIX}

The auxiliary elastic constants $C_{i}(i=1,2,3,4)$ are expressed as follows:

$$
\begin{aligned}
& C_{1}=n\left(1-n \mu_{v}^{2}\right) \\
& C_{2}=m\left(1+\mu_{h}\right)\left(1-\mu_{h}-2 n \mu_{v}^{2}\right) \\
& C_{3}=\left(1+\mu_{h}\right)\left\{n \mu_{v}+m\left(1-\mu_{h}-2 n \mu_{v}^{2}\right)\right\} \\
& C_{4}=1-\mu_{h}^{2}
\end{aligned}
$$

where

$$
n=E_{h} / E_{v}, m=G_{v} / E_{v}
$$

$E_{v}=$ Young's modulus of soil skeleton in the vertical direction

$E_{h}=$ Young's modulus of soil skeleton in horizontal directions

$G_{v}=$ shear modulus in vertical plane

$\mu_{v}=$ vertical Poisson's ratio of soil skeleton relating an imposed vertical strain to the resulting horizontal strain

$\mu_{h}=$ horizontal Poisson's ratio of soil skeleton relating an imposed horizontal strain to the resulting horizontal strain at right angles to the imposed strain

Under the present condition of $C_{1}=C_{4}=C_{2}+C_{3}$, two of these five elastic constants are restricted.

\section{REFERENCES}

1) Biot, M. A. : Theory of elasticity and consolidation for a porous anisotropic solid, Journ. Appl. Phys., Vol. 26, pp. 182 185, 1955.

2) Gazetes, G. : Strip foundations on a cross-anisotropic soil layer subjected to dynamic loading, Geotechnique, Vol. 31, pp. 161 179, 1981.

3) Milovic, D. M. and Touzot, G. : Bi-dimensional stress distribution in an anisotropic layer of finite thickness, Geotechnique, Vol. 20, pp. 198 203, 1970.

4) Murakami, Y. : Consolidation of a clay deposit with transversely isotropic permeability, Proc. JSCE., Vol. 326, pp. 73 82, 1982 (in Japanese).

5) Tamura, T. : Analysis of consolidation in anisotropic ground with sand drain, Proc. JSCE., Vol. 338, pp. 115 121, 1983 (in Japanese).

6) Yamaguchi, H. and Murakami, Y. : Plane strain consolidation of a clay layer with finite thickneess, Soils and Foundations, Vol. 16, No. 3, pp. $67 \sim 79,1976$. 